\title{
The Relationship between Language Ability and Cognitive Function in Patients with Schizophrenia
}

\author{
Sung-Jin Kim¹, Joo-Cheol Shim², Bo-Geum Kong', Je-Wook Kang', Jung-Joon Moon', Dong-Wook Jeon', \\ Sung-Soo Jung ${ }^{3}$, Beom-Joo Seo ${ }^{4}$, Do-Un Jung \\ ${ }^{1}$ Department of Psychiatry, Inje University Busan Paik Hospital, ${ }^{2}$ Shim Joo Cheol Psychiatry Clinic, ${ }^{3}$ Department of Psychiatry, Sharing \\ and Happiness Hospital, ${ }^{4}$ Department of Psychiatry, Busan Metropolitan Mental Hospital, Busan, Korea
}

\begin{abstract}
Objective: Cognitive dysfunction is common in people with schizophrenia, and language disability is one of the most notable cognitive deficits. This study assessed the use and comprehension ability of the Korean language in patients with schizophrenia and the correlations between language ability and cognitive function.

Methods: Eighty-six patients with schizophrenia and a group of 29 healthy controls were recruited. We assessed both clinical symptoms and cognitive functions including Korean language ability. For clinical symptoms, the Positive and Negative Syndrome Scale, Clinical Global Impression-Schizophrenia Scale, and Social and Occupational Functioning Assessment Scale were used. For the Korean language ability assessment, a portion of the Korean Broadcasting System (KBS) Korean Language Test was used. The Short-form of Korean-Wechsler Adult Intelligence Scale, the Korean version of the University of California San Diego (UCSD) Performance-based Skills Assessment (K-UPSA), and the Wisconsin Card Sorting Test (WCST) were used to assess cognitive functions.

Results: Schizophrenic patients had significantly lower scores in the language and cognitive function tests both in the total and subscale scores. Various clinical scores had negative correlations with reading comprehension ability of the KBS Korean Language Test. The WCST and a part of the K-UPSA had positive correlations with multiple domains of the language test. Conclusion: A significant difference was found between schizophrenic patients and controls in language ability. Correlations between Korean language ability and several clinical symptoms and cognitive functions were demonstrated in patients with schizophrenia. Tests of cognitive function had positive correlations with different aspects of language ability.
\end{abstract}

KEY WORDS: Cognition; Language; Schizophrenia

\section{INTRODUCTION}

Schizophrenia is a major mental illness with a prevalence rate of $1 \%$ in the adult population worldwide. Cognitive deficits are common symptoms in people with schizophrenia; $90 \%$ of patients have clinically relevant deficits at least in one cognitive domain and $75 \%$ show deficits in two or more cognitive domains. ${ }^{1)}$ Such a wide range of cognitive deficits appear gradually after disease onset and impair patients' functional reintegration. ${ }^{2,3)}$

Language disability is one of the most notable cognitive deficits in patients with schizophrenia. The two main components of language function are production (usage)

\footnotetext{
Received: March 30, 2015 / Revised: June 30, 2015

Accepted: July 27, 2015

Address for correspondence: Do-Un Jung, MD, PhD

Department of Psychiatry, Inje University Busan Paik Hospital, 75

Bokji-ro, Busanjin-gu, Busan 47392, Korea

Tel: +82-51-890-6189, Fax: +82-51-894-2532

E-mail: gabriel.jdu@gmail.com
}

and comprehension. Disability of verbal communication is a main symptom in the diagnosis of schizophrenia and usually appears in the form of thought disorder. Moreover, these language disabilities allow anticipating defects in social and occupational abilities. ${ }^{4}$ Impaired language comprehension usually manifests as an inability to understand figurative usage of language. Therefore, patients with schizophrenia exhibit difficulties in understanding proverbs, words with ambiguous meanings, and grammatically complex and long sentences. ${ }^{5)}$

Among several neurocognitive domains, verbal learning and memory show the most pronounced deficits compared to other cognitive domains in patients with schizophrenia; they also have the highest variability. ${ }^{6}$ Some studies showed the strong association of verbal memory impairment and initial work function in the patients with schizophrenia. ${ }^{7}$ Furthermore, deficits in neuropsychological tests with linguistic components are found in both chronic schizophrenic patients and children at risk of de-

(c) This is an Open-Access article distributed under the terms of the Creative Commons Attribution Non-Commercial License (http://creativecommons.org/licenses/by-nc/4.0) which permits unrestricted non-commercial use, distribution, and reproduction in any medium, provided the original work is properly cited. 
veloping schizophrenia ${ }^{8}{ }^{8}$ in early stages of schizophrenia ${ }^{9)}$ and at disease onset. ${ }^{10)}$ Of the two main theories of language disability of schizophrenia, the first describes the structural and functional abnormalities of semantic memory ${ }^{11)}$ and the second consists of abnormalities in the creation and usage of context due to impairments in working memory or executive function. ${ }^{12)}$

Several neuropsychological tests are used to examine cognitive functions in schizophrenic patients, but it has not been clearly established how improvement in tests of cognitive function relate to everyday social function improvement. Neuropsychological tests explain only about $25-50 \%$ of everyday function. ${ }^{13)}$ Occupational and social activities in everyday life do not only rely on cognitive function but also on a complex combination of motivation, desires, and environmental factors. The University of California San Diego (UCSD) Performance-based Skills Assessment (UPSA), which is highly sensitive and specific, was developed to evaluate everyday cognitive function in patients with schizophrenia. ${ }^{14)}$ It is a widely used role-playing tool that assess basic everyday living skills.

The neuropsychological tests used for evaluating cognitive function in schizophrenic patients in Korea typically do not include a tool to systematically evaluate ability of language usage. There are currently no tests to evaluate Korean language ability in everyday life for the schizophrenic patients. Furthermore, studies of Korean language ability in schizophrenic patients are very scarce. Recently, a Korean language test has been used by a few authorized institutes as an examination tool to measure universal Korean language ability in everyday life for the public. The Korean Broadcasting System (KBS) Korean Language Test is the nationally authorized language test to evaluate various language usage areas and has been continuously implemented since 2004. Therefore, the authors designed this study to evaluate the usefulness and the applicability of the KBS Korean Language Test to measure functional ability of language use in everyday life for schizophrenic patients, and to investigate correlations between language ability and other cognitive function.

\section{METHODS}

\section{Participants}

This study included patients who were diagnosed with schizophrenia using the Diagnostic and Statistical Manual of Mental Disorders 4th edition (DSM-IV) ${ }^{15)}$ and who met the following additional inclusion criteria: (1) between 18 and 60 years of age, (2) no changes in medication for the past three months, (3) relatively stable maintenance of symptoms, and (4) in an out-patient hospital or vocational rehabilitation. Exclusion criteria included were (1) an intelligence quotient (IQ) below 70 according to the short-form of the Korean-Wechsler Adult Intelligence Scale (K-WAIS), (2) a history of substance abuse or dependency according to DSM-IV criteria, and (3) a medical history of brain damage or neurological disorder. The control group was recruited from healthy persons who worked as volunteer caregivers for patients with psychiatric disorders at a hospital and were similar in age and educational background to the patient group. All subjects gave written informed consent. This study was approved by the institutional review board of Inje University Busan Paik Hospital, Busan, Korea (IRB: 11-032).

For the patient group, sex, age, level of education, duration of illness, and kind and dosage of current medications were assessed through interview and review of medical records. The dosage of antipsychotic drugs was converted into a chlorpromazine equivalent dose. ${ }^{16,17)}$

\section{Clinical Assessments}

For the evaluation of clinical symptoms, the Positive and Negative Syndrome Scale (PANSS) ${ }^{18)}$ the Clinical Global Impression-Schizophrenia scale (CGI-SCH), ${ }^{19)}$ and the Social and Occupational Functioning Assessment Scale (SOFAS) ${ }^{20)}$ were administered by psychiatrists.

The PANSS consists of 30 items in three subscales: 7 assessing positive symptoms; 7 , negative symptoms; and 16 , general psychopathology. Scores for each item range from 1 to 7 points and are compared to evaluation standards; higher scores indicate more severe psychopathology. The Korean version of the PANSS ${ }^{21)}$ was used. The CGI-SCH was developed to evaluate the general function of patients with schizophrenia via a short and standardized method by a psychiatrist. This scale evaluates 4 domains: positive, negative, depressive, and cognitive symptoms. The SOFAS evaluates the degree of a patient's social and vocational functions on a continuum ranging from 100 (optimal) to 1 (serious impairment).

\section{Neuropsychological Assessments}

\section{Short form of Korean-Wechsler Adult Intelligence Scale} (K-WAIS)

The K-WAIS is the Korean version of Wechsler Adult Intelligence Scale-Revised (WAIS-R) and evaluates verbal and performance intelligence. This study used a short form consisting of the picture completion, arithmetic, dig- 
it symbol, and similarities sub-tests of the K-WAIS. In a study analyzing the usefulness of WAIS-R short forms in schizophrenia, the short version consisting of these four sub-tests proved easy to perform compared to other short forms, and showed a high correlation between estimated intelligence and WAIS-R-tested intelligence. ${ }^{22)}$

The Korean version of the UCSD Performance-based Skills Assessment (K-UPSA)

The UCSD Performance-based Skills Assessment (UPSA) was developed to evaluate a patient's function in everyday life and evaluate basic livelihood skills through role-play tasks similar to those necessary for independent functioning in everyday life. ${ }^{14)}$ This tool consists of 5 functional domains: financial, communication, comprehension/planning, transportation, and household skills (estimated evaluation time: 30 minutes). The authors received approval from the copyright holder and used a standardized Korean version of the UPSA.

Wisconsin Card Sorting Test (WCST): Computer Version 4 The original WCST consists of 128 cards categorized by a combination of four kinds of color, shape, and number. The subjects first acquire the concept of the different categories, and without special instructions during the test, they have to discover the sorting principle (e.g., by color) that changes throughout the test. The WCST is a typical test that measures executive functions, including conceptual flexibility in response to feedback. In this study, the computerized WCST CV4 software program (Psychological Assessment Resources, Odessa, FL, USA) ${ }^{23)}$ was used. Common outcomes are Categories Achieved, Perseverative Errors, Perseverative Responses, total errors, and number of attempts to achieve the first category.

\section{Korean Broadcasting System (KBS) Korean Language Test}

The KBS Korean Language Test is a language ability qualification test authenticated by the Korean government. The KBS Korean Language Test evaluates effective and fluent language abilities as well as accurate and creative language abilities reflecting actual lingual circumstances, which can be easily reproduced in everyday life. Language ability is broadly classified into five domains, and each domain includes a variety of subfields: grammar ability, which includes vocabulary and grammar; comprehension ability, which includes listening comprehension and reading comprehension; expression ability, which includes writing and speaking; originality ability, which includes creative language use; and language culture ability, which consists of comprehensive knowledge related to language.

For this study, we obtained approval to edit and use parts of the KBS Korean Language Test from the KBS Korean language promotion director. Among the entire set of evaluation domains, listening comprehension, reading comprehension, and creative language use abilities were considered further. Based on the analysis of test questions 17-20, we pre-selected questions that yielded over $90 \%$ correct answers, and further included some questions with over $80 \%$ correct answers to balance the level of difficulty, resulting in 30 questions altogether. This procedure selected relatively simple items that did not require specific test preparations. The test was conducted in two sessions with 15 questions each to minimize fatigue.

\section{Statistical Methods}

Averages, standard deviations, and ranges were calculated for the continuous variables, and frequencies and percentages for discrete variables. Chi-square test, $t$-test, analysis of covariance (ANCOVA), and Wilcoxon rank sum test were used as statistical tests. Correlation analyses identified relationships between clinical symptoms and cognitive functions. The SAS 9.3 package (SAS Institute Inc., Cary, NC, USA) was used for analysis, and the significance level for all tests was set at $p<0.05$.

\section{RESULTS}

\section{Demographic Data}

The patient group consisted of 86 patients with schizophrenia and the control group, of 29 age-matched volunteers. Demographic and clinical characteristics are displayed in Table 1. The patients consisted of 50 men and 36 women (average age, $37.59 \pm 10.04$ years) and the control group, of 14 men and 15 women (average age, 37.34 \pm 9.15 years). The level of education for the patient and control groups was $13.03 \pm 1.58$ and $13.12 \pm 1.39$ years respectively. The average duration of illness for the patient group was $174.21 \pm 112.96$ months, and the dosage of antipsychotic drugs was $610.88 \pm 339.24 \mathrm{mg} /$ day, converted into a chlorpromazine-equivalent dose.

The total score of the PANSS was $61.76 \pm 19.63$, and the subscores were $15.04 \pm 6.25$ (positive subscale), 15.24 \pm 6.29 (negative subscale), and 31.49 \pm 9.65 (general psychopathology). The score for the CGI-SCH was $2.93 \pm 1.00$, and for the SOFAS was $52.77 \pm 11.44$. 
Table 1. Demographic and clinical characteristics of patients with schizophrenia and controls

\begin{tabular}{|c|c|c|c|}
\hline Characteristic & Schizophrenic $(n=86)$ & Control $(n=29)$ & $p$ value \\
\hline \multicolumn{4}{|l|}{ Gender } \\
\hline Male & $50(58.14)$ & $14(48.28)$ & \\
\hline Female & $36(41.86)$ & $15(51.72)$ & \\
\hline Age $(y r)$ & $37.59 \pm 10.04$ & $37.34 \pm 9.15$ & 0.907 \\
\hline Education (yr) & $13.03 \pm 1.58$ & $13.12 \pm 1.39$ & 0.093 \\
\hline K-WAIS & $92.31 \pm 12.18$ & $108.79 \pm 11.76$ & $<0.001$ \\
\hline Duration of illness (mo) & $174.21 \pm 112.96$ & & \\
\hline Average daily neuroleptic dose (mg), CPZE & $610.88 \pm 339.24$ & & \\
\hline \multicolumn{4}{|l|}{ PANSS } \\
\hline Positive subscale & $15.04 \pm 6.25$ & & \\
\hline Negative subscale & $15.24 \pm 6.29$ & & \\
\hline General psychopathology subscale & $31.49 \pm 9.65$ & & \\
\hline Total score & $61.76 \pm 19.63$ & & \\
\hline \multicolumn{4}{|l|}{ CGI-SCH } \\
\hline Positive symptoms & $3.17 \pm 1.31$ & & \\
\hline Negative symptoms & $2.44 \pm 1.21$ & & \\
\hline Depressive symptoms & $2.00 \pm 1.03$ & & \\
\hline Cognitive symptoms & $2.55 \pm 1.25$ & & \\
\hline Overall severity & $2.93 \pm 1.00$ & & \\
\hline SOFAS & $52.77 \pm 11.44$ & & \\
\hline
\end{tabular}

Values are presented as number (\%) or mean \pm standard deviation.

K-WAIS, Short-form of Korean-Wechsler Adult Intelligence Scale; CPZE, chlorpromazine equivalent; PANSS, Positive and Negative Syndrome Scale; CGI-SCH, Clinical Global Impression-Schizophrenia scale; SOFAS, Social and Occupational Functioning Assessment Scale.

Table 2. Comparison of patients with schizophrenia and controls in WCST, the KBS Korean Language Test and K-UPSA in which the effect of estimated $1 Q$ was controlled

\begin{tabular}{|c|c|c|c|c|}
\hline Variable & Schizophrenic $(n=86)$ & Control $(n=29)$ & F value & $p$ value \\
\hline \multicolumn{5}{|l|}{ WCST } \\
\hline PR & $39.37 \pm 26.75$ & $24.88 \pm 17.03$ & 3.82 & 0.054 \\
\hline PE & $32.47 \pm 19.52$ & $21.25 \pm 12.88$ & 4.51 & 0.037 \\
\hline CA & $1.49 \pm 1.88$ & $3.88 \pm 2.33$ & 12.30 & 0.001 \\
\hline \multicolumn{5}{|l|}{ KBS Korean Language Test } \\
\hline Listening comprehension & $4.77 \pm 2.70$ & $8.50 \pm 0.97$ & 9.42 & 0.003 \\
\hline Reading comprehension & $4.55 \pm 2.72$ & $9.40 \pm 1.07$ & 16.63 & $<0.001$ \\
\hline Creativity & $3.38 \pm 2.61$ & $8.80 \pm 1.03$ & 28.61 & $<0.001$ \\
\hline Total score & $12.70 \pm 6.99$ & $26.70 \pm 1.57$ & 23.67 & $<0.001$ \\
\hline \multicolumn{5}{|l|}{ K-UPSA } \\
\hline Financial & $15.86 \pm 3.86$ & $19.68 \pm 0.71$ & 4.71 & 0.032 \\
\hline Communication & $10.68 \pm 4.36$ & $16.73 \pm 1.60$ & 19.45 & $<0.001$ \\
\hline Comprehension/planning & $9.42 \pm 4.23$ & $16.02 \pm 2.94$ & 26.62 & $<0.001$ \\
\hline Transportation & $13.98 \pm 4.76$ & $19.52 \pm 1.11$ & 9.85 & $<0.001$ \\
\hline Household skills & $15.76 \pm 6.88$ & $19.82 \pm 0.94$ & 2.01 & $<0.001$ \\
\hline Total score & $65.69 \pm 17.48$ & $91.77 \pm 3.98$ & 20.25 & $<0.001$ \\
\hline
\end{tabular}

Values are presented as mean \pm standard deviation.

WCST, Wisconsin Card Sorting Test: PR, Perseverative Responses; PE, Perseverative Errors; CA, Numbers of Categories Achieved: KBS Korean Language Test, Korean Broadcasting System Korean Language Test; K-UPSA, Korean version of the University of California San Diego (UCSD) Performance-based Skills Assessment.

\section{Neuropsychological Assessment}

Results for the neuropsychological and language assessments are summarized in Table 2. On the WCST, the patient group had $39.37 \pm 26.75$ and the controls, $24.88 \pm 17.03$ Perseverative Responses $(p=0.054)$; the patient group had $32.47 \pm 19.52$ and the controls, $21.25 \pm 12.88$ Perseverative Errors ( $p=0.037$ ); and the patient group scored $1.49 \pm 1.88$ and the controls, $3.88 \pm 2.33$ in the Categories Achieved $(p=0.001)$.

For the KBS Korean Language Test, the patients' average score was $12.70 \pm 6.99$, and the controls' average score was $26.70 \pm 1.57$, yielding a significant difference $(p<$ $0.001)$. This difference also manifested in the specific domains of listening comprehension, reading comprehension, and creativity, with the patient group achieving scores of $4.77 \pm 2.70,4.55 \pm 2.72$, and $3.38 \pm 2.61$, respectively, and the 
control group achieving scores of $8.50 \pm 0.97,9.40 \pm 1.07$, and $8.80 \pm 1.03$, respectively. Differences for the subdomains were also statistically significant (listening comprehension, $p=0.003$; other subdomains, $p<0.001$ ).

The total score of the K-UPSA for the patient group was $65.69 \pm 17.48$ and that for the control group was $91.77 \pm$ 3.98. The patient group showed a significantly lower performance on the total score and on all subdomains (financial domain, $p=0.032$; other subdomains, $p<0.001$ ).

\section{Correlation between KBS Korean Language Test and Clinical Symptoms}

The performance in the KBS Korean Language Test correlated with aspects of clinical symptoms in the current sample of patients with schizophrenia (Table 3). Although age, education level, and medication did not correlate with performance in the KBS Korean Language Test, the duration of illness was shown to have a significant negative correlation with the total score. IQ, as assessed by the short form of the K-WAIS, was found to have a positive correlation with the total score $(p=0.017)$, and with the reading comprehension $(p=0.022)$ and listening comprehension ( $p=0.008)$ domains, but not with the creativity domain.

The total score and all subscales of the PANSS had negative correlations with the reading comprehension domain (total score, $p=0.002$; positive subscale, $p=0.003$; negative

Table 3. Correlations between the KBS Korean Language Test and clinical symptoms in patients with schizophrenia

\begin{tabular}{|c|c|c|c|c|c|c|c|c|}
\hline \multirow{2}{*}{ Variable } & \multicolumn{2}{|c|}{ Listening comprehension } & \multicolumn{2}{|c|}{ Reading comprehension } & \multicolumn{2}{|c|}{ Creativity } & \multicolumn{2}{|c|}{ Total score } \\
\hline & $r$ & $p$ & $r$ & $p$ & $r$ & $p$ & $r$ & $p$ \\
\hline Age & -0.167 & 0.220 & -0.120 & 0.377 & -0.140 & 0.305 & -0.157 & 0.248 \\
\hline Education & 0.140 & 0.304 & 0.079 & 0.562 & 0.148 & 0.278 & 0.148 & 0.277 \\
\hline Duration of illness & -0.246 & 0.068 & -0.238 & 0.078 & -0.247 & 0.067 & -0.276 & $0.040^{*}$ \\
\hline CPZ equivalent & -0.138 & 0.309 & -0.143 & 0.294 & -0.046 & 0.739 & -0.164 & 0.227 \\
\hline K-WAIS & 0.328 & $0.022^{*}$ & 0.378 & $0.008^{* *}$ & 0.193 & 0.184 & 0.341 & $0.017^{*}$ \\
\hline \multicolumn{9}{|l|}{ PANSS } \\
\hline Positive & -0.110 & 0.419 & -0.388 & $0.003^{* *}$ & -0.099 & 0.468 & -0.253 & 0.060 \\
\hline Negative & -0.059 & 0.666 & -0.333 & $0.012^{*}$ & -0.060 & 0.662 & -0.162 & 0.233 \\
\hline General & -0.003 & 0.985 & -0.307 & $0.021^{*}$ & -0.025 & 0.857 & -0.125 & 0.360 \\
\hline Total & -0.072 & 0.600 & -0.400 & $0.002^{* *}$ & -0.074 & 0.589 & -0.209 & 0.122 \\
\hline \multicolumn{9}{|l|}{ CGI-SCH } \\
\hline Positive symptoms & -0.159 & 0.241 & -0.256 & 0.057 & -0.195 & 0.151 & -0.258 & 0.055 \\
\hline Negative symptoms & 0.030 & 0.829 & -0.264 & $0.049^{*}$ & -0.085 & 0.535 & -0.092 & 0.500 \\
\hline Depressive symptoms & -0.185 & 0.172 & -0.317 & $0.017^{*}$ & -0.192 & 0.156 & -0.270 & $0.044^{*}$ \\
\hline Cognitive symptoms & -0.246 & 0.067 & -0.488 & $<0.001^{\star \star \star}$ & -0.244 & 0.070 & -0.377 & $0.004^{* *}$ \\
\hline Overall severity & -0.231 & 0.086 & -0.418 & $0.001^{* *}$ & -0.185 & 0.172 & -0.339 & $0.011^{*}$ \\
\hline SOFAS & 0.112 & 0.411 & 0.301 & $0.024^{*}$ & 0.045 & 0.743 & 0.167 & 0.218 \\
\hline
\end{tabular}

CPZ, chlorpromazine; K-WAIS, Short-form of Korean-Wechsler Adult Intelligence Scale; PANSS, Positive and Negative Syndrome Scale; CGI-SCH, Clinical Global Impression-Schizophrenia scale; SOFAS, Social and Occupational Functioning Assessment Scale.

${ }^{*} p<0.05 ;{ }^{* *} p<0.01 ;{ }^{* * *} p<0.001$.

Table 4. Correlations between the KBS Korean Language Test and cognitive functions in patients with schizophrenia

\begin{tabular}{|c|c|c|c|c|c|c|c|c|}
\hline \multirow{2}{*}{ Variable } & \multicolumn{2}{|c|}{ Listening comprehension } & \multicolumn{2}{|c|}{ Reading comprehension } & \multicolumn{2}{|c|}{ Creativity } & \multicolumn{2}{|c|}{ Total score } \\
\hline & $r$ & $p$ & $r$ & $p$ & $r$ & $p$ & $r$ & $p$ \\
\hline \multicolumn{9}{|l|}{ WCST } \\
\hline PR & -0.147 & 0.318 & 0.129 & 0.381 & -0.158 & 0.284 & -0.065 & 0.659 \\
\hline PE & -0.160 & 0.278 & 0.112 & 0.448 & -0.167 & 0.258 & -0.083 & 0.576 \\
\hline CA & 0.331 & $0.021^{*}$ & 0.248 & 0.090 & 0.388 & $0.007^{* *}$ & 0.395 & $0.006^{* *}$ \\
\hline \multicolumn{9}{|l|}{ K-UPSA } \\
\hline Financial & 0.368 & $0.005^{\star *}$ & 0.491 & $<0.001^{* * *}$ & 0.197 & 0.147 & 0.405 & $0.002^{* *}$ \\
\hline Communication & 0.451 & $0.001^{* *}$ & 0.432 & $0.001^{* *}$ & 0.332 & $0.013^{*}$ & 0.494 & $<0.001^{* * *}$ \\
\hline Comprehension/Planning & 0.083 & 0.542 & -0.079 & 0.564 & 0.097 & 0.475 & 0.039 & 0.775 \\
\hline Transportation & 0.429 & $0.001^{\star *}$ & 0.543 & $<0.001^{* * *}$ & 0.316 & $0.018^{*}$ & 0.524 & $<0.001^{* *}$ \\
\hline Household skills & 0.204 & 0.131 & 0.191 & 0.160 & 0.173 & 0.203 & 0.202 & 0.136 \\
\hline Total score & 0.458 & $<0.001^{\star \star *}$ & 0.470 & $<0.001^{* * *}$ & 0.346 & $0.001^{* *}$ & 0.500 & $<0.001^{* * *}$ \\
\hline
\end{tabular}

WCST, Wisconsin Card Sorting Test; PR, Perseverative Responses; PE, Perseverative Errors; CA, Categories Achieved; K-UPSA, the Korean version of the University of California San Diego (UCSD) Performance-based Skills Assessment.

${ }^{\star} p<0.05 ;{ }^{* *} p<0.01 ;{ }^{* \star *} p<0.001$. 
subscale, $p=0.012$; general psychopathology subscale, $p=0.021$ ). For the CGI-SCH, the positive-symptoms subscale did not correlate with performance on the KBS Korean Language Test; however, the negative-symptoms subscale correlated negatively with the reading comprehension domain $(p=0.049)$. Depressive symptoms, cognitive symptoms, and overall severity in the CGI-SCH had negative correlations with the reading comprehension domain $(p=0.017, p<0.001, p=0.001)$ and total score ( $p=0.044, p=0.004, p=0.011$ ). The SOFAS was shown to have a positive correlation with the reading comprehension domain of the KBS Korean Language Test $(p=0.024)$.

\section{Correlation between KBS Korean Language Test and Cognitive Function}

In the WCST, Perseverative Responses and Perseverative Errors were not correlated with result of the KBS Korean Language Test (Table 4), but numbers of Categories Achieved were correlated positively with listening comprehension $(p=0.021)$, creativity $(p=0.007)$, and total score $(p=0.006)$.

The K-UPSA correlated with results of the KBS Korean Language Test in various domains (Table 4). The total score of K-UPSA was correlated positively with all fields (reading comprehension, listening comprehension, creativity, and total score) of the KBS Korean Language Test. The comprehension/planning and household skills subscales did not correlate with language ability. Communication and transportation subscales were positively correlated with all language domains, but the financial subscale did not correlate with the creativity domain.

\section{DISCUSSION}

This study aimed to measure Korean language use and comprehension ability in everyday life in patients with schizophrenia and to investigate the correlation between Korean language ability and cognitive function. A portion of the KBS Korean Language Test was used for language assessment, the short form of K-WAIS and the WCST measured cognitive functions, and the K-UPSA assessed cognitive functions in everyday life.

Although the patient group and controls were matched for age and educational background, the control group had a higher IQ than the patient group, as measured with the short form of the K-WAIS. This result matches various studies assessing intelligence in patients with schizophrenia, who consistently show lower intelligence scores than normal controls over a range of periods from pre- morbid and onset of schizophrenia ${ }^{24)}$ to the first episode of schizophrenia, ${ }^{25)}$ and after progression of the disease. ${ }^{26)}$

The KBS Korean Language Test assessed the use and comprehension ability of Korean language in everyday life, and we selected questions with a percentage of correct answers above $90 \%$ (to adjust the difficulty, some questions with a percentage above $80 \%$ were also chosen) to assess everyday abilities more easily. The results of the control group were similar to those of participants in the original KBS Korean Language Test study. To measure language ability of patients with schizophrenia, several methods have been applied in previous studies; the vocabulary test included in the WAIS-R, the Boston Naming Test, language proficiency tests, and other tools were mainly used to measure vocabulary. ${ }^{25,27)}$ Reading comprehension ability, that is, how accurately words are read, was measured with a reading comprehension test of the Wide Range Achievement Test. Since the KBS Korean Language Test assesses how accurately subjects can understand language using advertising descriptions and announcements, it more accurately reflects the language ability of everyday life. Since vocabulary forms the basis of language ability, the assessment of vocabulary ability is necessary to reliably assess language ability in schizophrenic patients. Although many studies focus on basic language ability, only a few reproduced the use of language in everyday life. $^{28,29)}$

The performance of schizophrenic patients in this study was poorer than that of the control group in the listening comprehension, reading comprehension, and creativity domains. Age and education level had no correlation with language ability, whereas the results of the intelligence test had a high correlation with measures of language ability.

Measurements of clinical symptoms using the PANSS and the CGI-SCH mainly correlated with the reading comprehension domain and did not show much association with the listening comprehension and creativity domains. This result is difficult to interpret based on the current data and requires additional studies that specifically relate listening comprehension and reading comprehension skills of schizophrenic patients to the clinical symptoms.

The patient group showed poorer performance than the control group on the WCST and the K-UPSA, which both assessed cognitive functions. These results were similar to those of existing studies. ${ }^{14,27)}$ Executive functions measured with the WCST correlated with Korean language abilities. The correlation between executive function and language comprehension ability has been described by another study. ${ }^{30)}$ However, this previous study mainly used 
the interpretation of proverbs or words with dual meaning to assess language comprehension, while the current study mainly used advertising descriptions, announcements, or instructions in which conveyance of the meaning is comparatively specific and clear.

The UPSA is a tool that measures the cognitive function of patients through role-play, by setting up a situation that can occur in everyday life. ${ }^{14)}$ The K-UPSA showed a positive correlation with the KBS Korean Language Test in most of the domains. In accordance with the intended objective of this study, this can be interpreted as a relationship between language deficiency and the decline of cognitive function in everyday life. If treatments that can improve language ability are added to therapeutic programs aimed at improving cognitive functions, we can expect an overall improvement in cognitive functions in schizophrenic patients. Moreover, tests of language ability could be used as instruments for assessing the effects of a cognitive function treatment program.

This study has some limitations. First, the KBS Korean Language Test is not specifically developed for the assessment of language ability in schizophrenic patients. Moreover, the questions used for the Korean language ability assessment are limited to this study and cannot be used in other studies. Therefore, the development of a standardized assessment instrument is needed to accurately assess Korean language ability in schizophrenic patients. Second, although the age and education level of both groups were about the same, the measured intelligence of the patient group was significantly lower than that of the control group. This difference can affect the results of cognitive function tests; therefore, we should be cautious in comparing cognitive functions between patient and control groups. Third, medications taken by the schizophrenic patients were not controlled. Despite the fact that many medications, including antipsychotics, can affect cognitive function, the influence of the medications was not assessed in this study.

A review of the relevant literature found this to be the first study assessing the Korean language ability of patients with schizophrenia in everyday life. Although no standardized test instruments were used, it was confirmed repeatedly that language deficiencies were strongly correlated with executive and cognitive functions used in everyday life. For future studies in Korea, we should consider language ability as a crucial factor when assessing cognitive function, especially in the everyday lives of schizophrenic patients, and as a useful method of determining the effects of cognitive rehabilitation on cognitive function. Thus, the development of a systematic and standardized test that can accurately assess Korean language ability in schizophrenic patients is needed for future studies and intervention.

\section{REFERENCES}

1. Palmer BW, Heaton RK, Paulsen JS, Kuck J, Braff D, Harris $\mathrm{MJ}$, et al. Is it possible to be schizophrenic yet neuropsychologically normal? Neuropsychology 1997;11:437-446.

2. Green MF. What are the functional consequences of neurocognitive deficits in schizophrenia? Am J Psychiatry 1996;153:321-330.

3. Kaneko Y, Keshavan M. Cognitive remediation in schizophrenia. Clin Psychopharmacol Neurosci 2012;10:125-135.

4. Bowie CR, Harvey PD. Communication abnormalities predict functional outcomes in chronic schizophrenia: differential associations with social and adaptive functions. Schizophr Res 2008;103:240-247.

5. Goldberg TE, Aloia MS, Gourovitch ML, Missar D, Pickar D, Weinberger DR. Cognitive substrates of thought disorder, I: the semantic system. Am J Psychiatry 1998;155:1671-1676.

6. Bowie CR, Harvey PD. Cognition in schizophrenia: impairments, determinants, and functional importance. Psychiatr Clin North Am 2005;28:613-633, 626.

7. Bryson G, Bell MD, Kaplan E, Greig T. The functional consequences of memory impairments on initial work performance in people with schizophrenia. J Nerv Ment Dis 1998;186:610-615.

8. Reichenberg A, Weiser M, Caspi A, Knobler HY, Lubin G, Harvey PD, et al. Premorbid intellectual functioning and risk of schizophrenia and spectrum disorders. J Clin Exp Neuropsychol 2006;28:193-207.

9. Simon AE, Cattapan-Ludewig K, Zmilacher S, Arbach D, Gruber K, Dvorsky DN, et al. Cognitive functioning in the schizophrenia prodrome. Schizophr Bull 2007;33:761-771.

10. Wood SJ, Tarnawski AU, Proffitt TM, Brewer WJ, Savage GR, Anderson V, et al. Fractionation of verbal memory impairment in schizophrenia and schizophreniform psychosis. Aust N Z J Psychiatry 2007;41:732-739.

11. Aloia MS, Gourovitch ML, Missar D, Pickar D, Weinberger DR, Goldberg TE. Cognitive substrates of thought disorder, II: specifying a candidate cognitive mechanism. Am J Psychiatry 1998;155:1677-1684.

12. Cohen JD, Servan-Schreiber D. Context, cortex, and dopamine: a connectionist approach to behavior and biology in schizophrenia. Psychol Rev 1992;99:45-77.

13. Harvey PD, Howanitz E, Parrella M, White L, Davidson M, Mohs RC, et al. Symptoms, cognitive functioning, and adaptive skills in geriatric patients with lifelong schizophrenia: a comparison across treatment sites. Am J Psychiatry 1998; 155:1080-1086.

14. Patterson TL, Goldman S, McKibbin CL, Hughs T, Jeste DV. UCSD Performance-Based Skills Assessment: development of a new measure of everyday functioning for severely mentally ill adults. Schizophr Bull 2001;27:235-245.

15. American Psychiatric Association. Diagnostic and statistical manual of mental disorders: DSM-IV. 4th ed. Washington, DC:American Psychiatric Association; 1994.

16. Rey MJ, Schulz P, Costa C, Dick P, Tissot R. Guidelines for the dosage of neuroleptics. I: Chlorpromazine equivalents of orally administered neuroleptics. Int Clin Psychopharmacol 1989;4:95-104.

17. Gardner DM, Murphy AL, O'Donnell H, Centorrino F, 
Baldessarini RJ. International consensus study of antipsychotic dosing. Am J Psychiatry 2010;167:686-693.

18. Kay SR, Fiszbein A, Opler LA. The positive and negative syndrome scale (PANSS) for schizophrenia. Schizophr Bull 1987;13:261-276.

19. Haro JM, Kamath SA, Ochoa S, Novick D, Rele K, Fargas $\mathrm{A}$, et al; SOHO Study Group. The Clinical Global Impression-Schizophrenia scale: a simple instrument to measure the diversity of symptoms present in schizophrenia. Acta Psychiatr Scand Suppl 2003;(416):16-23.

20. Goldman HH, Skodol AE, Lave TR. Revising axis $V$ for DSM-IV: a review of measures of social functioning. Am J Psychiatry 1992;149:1148-1156.

21. Yi JS, Ahn YM, Shin HK, An SK, Joo YH, Kim SH, et al. Reliability and validity of the Korean version of the positive and negative syndrome scale. $J$ Korean Neuropsychiatr Assoc 2001;40:1090-1105.

22. Allen DN, Huegel SG, Gurklis JA Jr, Kelley ME, Barry EJ, van Kammen DP. Utility of WAIS-R short forms in schizophrenia. Schizophr Res 1997;26:163-172.

23. Heaton RK. Wisconsin Card Sorting Test Manual. Rev. and expanded ed. Odessa, FL:Psychological Assessment Resources; 1993.
24. Carrión RE, Goldberg TE, McLaughlin D, Auther AM, Correll CU, Cornblatt BA. Impact of neurocognition on social and role functioning in individuals at clinical high risk for psychosis. Am J Psychiatry 2011;168:806-813.

25. Mesholam-Gately RI, Giuliano AJ, Goff KP, Faraone SV, Seidman LJ. Neurocognition in first-episode schizophrenia: a meta-analytic review. Neuropsychology 2009;23:315-336.

26. Morrison G, O'Carroll R, McCreadie R. Long-term course of cognitive impairment in schizophrenia. Br J Psychiatry 2006;189:556-557.

27. Yamashita C, Mizuno M, Nemoto T, Kashima H. Social cognitive problem-solving in schizophrenia: associations with fluency and verbal memory. Psychiatry Res 2005;134: 123-129.

28. Souchay C, Bacon E, Danion JM. Metamemory in Schizophrenia: an exploration of the feeling-of-knowing state. $J$ Clin Exp Neuropsychol 2006;28:828-840.

29. Haas MH, Chance SA, Cram DF, Crow TJ, Luc A, Hage S. Evidence of pragmatic impairments in speech and proverb interpretation in schizophrenia. J Psycholinguist Res 2015; 44:469-483.

30. Kuperberg GR. Language in schizophrenia Part 1: an Introduction. Lang Linguist Compass 2010;4:576-589. 